\title{
Design of Plant Growth Cabinet Control System Based on LabVIEW
}

\author{
Jiangtao Zou ${ }^{1, a}$, Liguo Tian ${ }^{2, b}$,Jianglin Wei, Meng Li, Zhiqi Liu
}

${ }^{1}$ Tianjin Key laboratory of Information Sensing \&Intelligent Control, Tianjin University of Technology and Education, Tianjin 300222 China

a 564710622@qq.com, ${ }^{b}$ tlg1234@163.com

Keyword: Virtual instrument; DAQ; VISA; Digital I/O

\begin{abstract}
For urban land resources are increasingly strained reality, the use of artificial controllable LED lights and virtual instrument technology, simulation of plant growth in natural environment as possible.This system of artificial plant growth platform controlled environment, focusing on the virtual instrument technology in plant growth cabinet measurement and control system, using virtual instrument software platform LabVIEW, a combination of hardware NI-PCI data acquisition card, using data acquisition (DAQ) technology, serial communication technology (VISA), digital I/O port technology to build software control platform, upload sensor data in real time via the data acquisition to the host computer, the host are displayed in real-time graphical interface to design and control of plant growth cabinet system that can achieve a controlled environment plant growth platform.
\end{abstract}

\section{Introduction}

With the development of the city, land resources are increasingly scarce, while in traditional plant cultivation, the land is an important integral part of the reality in this case, making it possible to artificial indoor cultivation of plants (such as flowers cultivation). With the rapid development of integrated circuits and computer communication technologies, virtual instrumentation technology emerges. Virtual instrument technology and control technology and computer technology is the product of combining powerful resource that makes use of computer hardware and software, reduce system cost, enhance system flexibility; usually more, in the form of lower machine composed of virtual instrumentation and control automation systems, which PC is usually composed by the PC, to achieve control of the lower machine and complete analysis of the data storage display functions; lower computer from the data acquisition circuit, data communication between the upper and lower machine commonly used RS-232C serial communication [1].

\section{The overall design of the system platform}

The system is based on NI (National Instruments) company's PCI-6014 data acquisition card and LabVIEW software platform, developed by plant growth control system, along with the rapid development of virtual instrument technology, can well meet the system requirements for achieving collection that plant growth and control system development becomes easy and efficient. LabVIEW as a means of virtual panel user interface and a block diagram of the establishment of a virtual instrument graphics programming, has been widely applied to industry, academia and research laboratories and other areas, it is regarded as a standard data acquisition and instrument control software instruments [2]. The system is based on LabVIEW software platform and PCI data acquisition cards that developed for plant growth cabinet and control system, including data acquisition, serial communication, data storage and other functions, to achieve dynamic monitoring plant growth inside the various environmental parameters. 


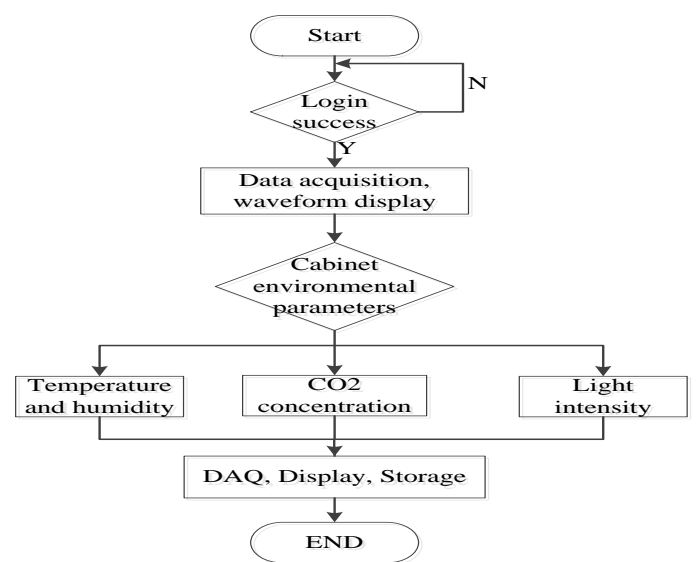

Fig. 1 The system flow chart

As shown in Fig. 1,after a successful login in the system, the PC started to show growth cabinet each data, the use of LabVIEW software platform to control the opening of each of the next crew to make the cabinet to achieve optimal dynamic environmental parameters in the PC display, save data Finally, exit the system.

\section{The System Hardware Design}

Due to the high PCI bus stability, multi-channel DAQ collection, transmission rate is high, small CPU occupancy rate, because of the PCI bus has these advantages, this monitoring and control system uses a cost-effective data acquisition card PCI-6014, which has 16 single-ended/8 differential analog input sampling rates up to $200 \mathrm{~K} / \mathrm{V}$, conversion accuracy is 16 , the input range from $( \pm 0.5)-( \pm 10) \mathrm{V}$, has eight $(5 \mathrm{~V} / \mathrm{TTL})$ digital I/O line, two Counter / Timers [3]. The system uses a multi-sensor acquisition technology, applied to the soil temperature and humidity, indoor temperature and humidity, light intensity, carbon dioxide and other sensors, through the acquisition card hardware part of the real-time sensor data uploaded to the host computer, and then show up in LabVIEW software platform program.

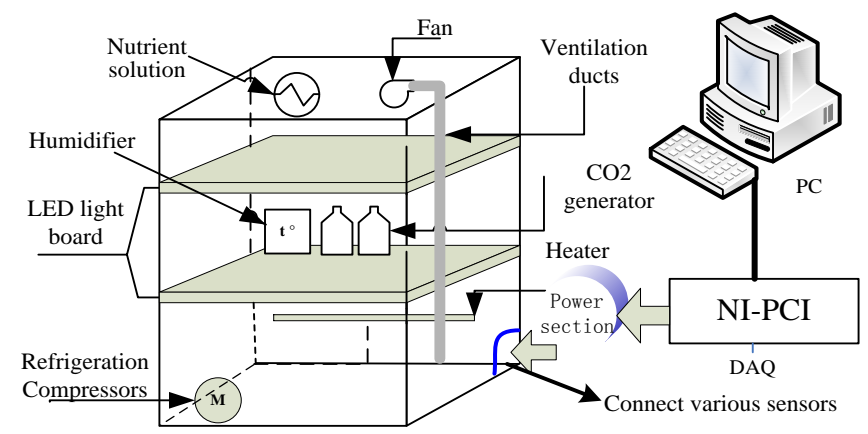

Fig. 2 The plant growth cabinet hardware

As shown in Fig. 2, access to a variety of sensors PCI-6014 data acquisition channel, wherein the data card digital I/O port and the sensors connected to the power supply module to achieve control bits fans, refrigeration compressors, humidifiers, intelligent light intensity under.

Type of power plant growth environment simulation systems are mainly: 220V power supply environment for the compressor in the refrigeration unit; $110 \mathrm{~V}$ power supply for environmental heating rods; $24 \mathrm{~V}$ power supply for soil temperature and humidity sensors, CO2 sensors, light sensors, LED light panels; $12 \mathrm{~V}$ power supply for heating and cooling control nutrient solution and carbon dioxide generator pressure pump and power supply[4].

\section{The System Software Design}

PC software design. Software design of the system is the core of the entire plant growth cabinet, but also an important part of the entire system. This paper uses a modular software design and hierarchical design, top-down design methodology, based on the general requirements and 
performance parameters of the system, the system is divided into various functional modules, the system consists DAQ data acquisition module, serial communication module, and digital I/O port modules, each module and then gradually divided into smaller sub-modules. This hierarchical modular structure of the program will not only increase the maintainability of the program, but also increases the readability of the program so that the program flow chart more clarity, while avoiding a lot of repeat programming.

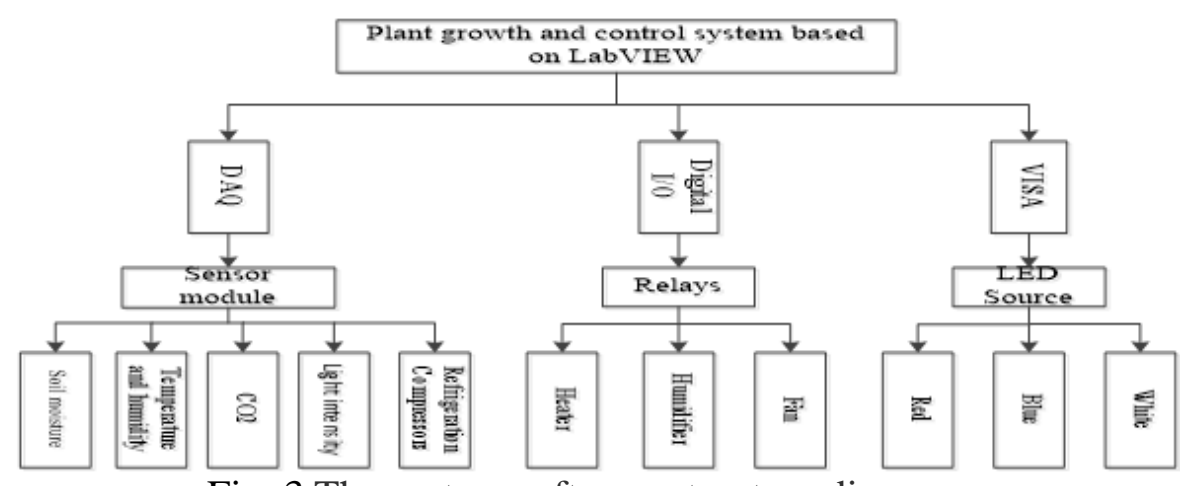

Fig. 3 The system software structure diagram

As shown in Fig. 3, System software mainly by DAQ, Digital I/O, VISA three modules. DAQ data acquisition module mainly uses Acquisition child controls in LabVIEW, using PCI-6014 five sampling channel, were collected in soil temperature and humidity, CO2 concentration, the growth temperature and humidity, light intensity. In the digital I/O port module, PCI-6014 data acquisition card with eight I/O port, the output for the 5V TTL level. In this paper, the acquisition card in three I/O ports, three relays are used to control the refrigeration compressors, heating rods, fans, and then to control plant growth cabinet hardware circuit through the relay, intelligent control plant growth cabinet environment. In the VISA serial communication module, using RS-232 and the lower position of the MCU communication, written communication protocol in the MCU, the PC sends commands to the MCU, and then the single-chip implementation of host computer's command, were used to control the red, blue, white LED lamp light intensity, PC machine so superior, only needs to operate the LabVIEW measurement and control software platform, you can control the LED lower light intensity.

Due to a variety of LabVIEW graphical provide drivers do not have to be familiar with the user to drive the PCI bus can be a variety of bus I/O interface device, the measured signal for input, output, data acquisition, amplification, A/D conversion, and further a computer for further analysis and processing. The plant growth cabinet with multi-channel monitoring system sensor data collected by the PC software displays real-time graphical interface, and can automatically monitoring the growth cabinet environmental data through the data analysis software platform, then under the control bit hardware circuit makes cabinet dynamic equilibrium temperature and humidity to ensure that the plant is always in the most appropriate environment to grow.

\section{PC LabVIEW program to achieve}

Entire program uses "event trigger" approach to writing. When the host computer to enter a user name and password correctly, the PC starts to initialize, after initialization is successful, the next bit of the sensor to start measuring the growth cabinet environment and uploaded to the host computer, the host computer system started to show growth cabinet environmental parameters in real time.

1)Data acquisition mode

LabVIEW is a graphical programming language written by NI-DAQ module, written procedures, can be well connected to the host computer and hardware together.

2)Serial communication mode

Using PC comes with RS-232 serial port, the PC serial communication program functions primarily through the serial port configuration that comes with LabVIEW VISA functions implemented. Run LabVIEW2010, create a VI, add a "serial configuration function" on the block 
diagram, and VISA resource name, baud rate, data bits, etc. terminals defined.

3)Digital I/O mode

Using PCI-6014 comes with 8 digital I/O mouth 3, respectively, as the relay input signal, a hardware circuit is shown, when the temperature is too low, start to control the heating rods digital $\mathrm{I} / \mathrm{O}$ ports, so that the cabinet the temperature rises, the same when the temperature is too high, open refrigeration compressor control digital I/O ports, quickly let the temperature drop inside the same cabinet when the $\mathrm{CO} 2$ concentration is too low or too high, start fan $\mathrm{I} / \mathrm{O}$ ports, this will allow the cabinet to achieve a dynamic balance, so that optimum plant growth.

\section{Conclusions}

Using PCI-6014 data acquisition card and existing laboratory equipment to establish a monitoring and control system for plant growth cabinet, the common good of the system, eliminating the operation of multi-sensor information collected some of the tedious work; Multi-sensor information is displayed on the same screen, so that measurement information more intuitive; formation of a unified data storage file format is conducive to post-processing of multi-sensor information is stored. The system takes full advantage of the flexibility of virtual instrumentation and multiple-input multiple-output characteristics of the computer, multi-sensor, LabVIEW software combine to form a virtual instrument system, multi-information monitoring and control of plant growth, further studies for future use virtual instrument and control system for plant growth as well as multi-sensor information fusion foundation.

\section{Acknowledgement}

This work is supported by the National High Technology Research and Development Program of China (863 Program, No.SS2013AA03120).

School-level Scientific Research Project of Tianjin University of Technology Education(KJ13-02).

\section{References}

[1] Zhi Jun Zhang, Xiao Li Xu. The LabVIEW and Wi-Fi technology in wireless data acquisition system based on [J]. Control and instruments in chemical industry, 2012:367-371. (In Chinese)

[2]Kozai Tetal. Commercialized closed system with artificial lighting for high quality plant production at low cost.2004 CIGR International Conference Beijing, 2004, 50: (6),238-245.

[3] Xi Hui Chen, Yin Hong Zhang. LabVIEW8.20 programming from entry to the master [M]. Beijing: Tsinghua University press, 2007. (In Chinese)

[4]Ohashi-Kaneko K,Matsuda R,Goto E,et al. Growth of rice plants under red light with or without supplemental blue light[J].Soil Science and Plant Nutrition, 2006 ,52(4): 444-452. 